\title{
Altered intrinsic regional brain spontaneous activity in patients with comitant strabismus: a resting-state functional MRI study
}

This article was published in the following Dove Press journal:

Neuropsychiatric Disease and Treatment

3 June 2016

Number of times this article has been viewed

\author{
Xin Huang ${ }^{1,2, *}$ \\ Sheng-Hong $\mathrm{Li}^{3, *}$ \\ Fu-Qing Zhou ${ }^{3}$ \\ Ying Zhang' \\ Yu-Lin Zhong' \\ Feng-Qin $\mathrm{Cai}^{3}$ \\ Yi Shao' \\ Xian-Jun Zeng ${ }^{3}$ \\ 'Department of Ophthalmology, \\ The First Affiliated Hospital of \\ Nanchang University, Jiangxi \\ Province Clinical Ophthalmology \\ Institute and Oculopathy Research \\ Centre, Nanchang, ${ }^{2}$ Department of \\ Ophthalmology, The First People's \\ Hospital of Jiujiang City, Jiujiang, \\ ${ }^{3}$ Department of Radiology, The First \\ Affiliated Hospital of Nanchang \\ University, Jiangxi Province Medical \\ Imaging Research Institute, Nanchang, \\ Jiangxi, People's Republic of China \\ *These authors contributed equally \\ to this work
}

Correspondence: Yi Shao

Department of Ophthalmology,

The First Affiliated Hospital of Nanchang

University, No 17, Yongwaizheng Street,

Donghu District, Nanchang 330006,

Jiangxi, People's Republic of China

Tel +86 79l 88692520

Email freebee99@163.com

Xian-Jun Zeng

Department of Radiology, The First

Affiliated Hospital of Nanchang

University, No 17, Yongwaizheng Street,

Donghu District, Nanchang 330006,

Jiangxi, People's Republic of China

Tel +86 791 88695132

Email375।35747@qq.com
Objective: To investigate the underlying regional homogeneity ( $\mathrm{ReHo}$ ) of brain-activity abnormalities in patients with comitant strabismus (CS) and their relationship with behavioral performance.

Methods: Twenty patients with CS (ten men and ten women) and 20 (ten men and ten women) age-, sex-, and education-matched healthy controls (HCs) underwent resting-state functional magnetic resonance imaging scans. The ReHo method was used to assess local features of spontaneous brain activities. Patients with CS were distinguished from HCs by receiver operating characteristic curve. Correlation analysis was performed to explore the relationship between the observed mean ReHo values of the different brain areas and behavioral performance.

Results: Compared to HCs, the patients with CS showed significantly increased ReHo values in the right inferior temporal cortex/fusiform gyrus/cerebellum anterior lobe, right lingual gyrus, and bilateral cingulate gyrus. We did not find any relationship between the observed mean ReHo values of the different brain areas and behavioral performance.

Conclusion: CS causes dysfunction in many brain regions, which may explain the fusion compensation in CS.

Keywords: comitant strabismus, regional homogeneity, functional magnetic resonance imaging, resting state

\section{Introduction}

Strabismus is an ocular alignment disorder characterized by a horizontal, vertical, and/or torsional deviation of one eye relative to the other, which may result in compromised binocular vision and amblyopia. ${ }^{1,2}$ Strabismus is classified roughly into comitant and incomitant types. According to a study conducted in Central China, strabismus was prevalent in $108(5.0 \%)$ out of the 2,363 eligible students. ${ }^{3}$ Clinically, strabismus also exhibits stereopsis impairment. At present, surgery is the major method of strabismus correction. ${ }^{4}$

Optimal eye movements and alignment require a functional oculomotor system. Extraocular muscle (EOM) dysfunction is an important factor in strabismus. Initially, it was reported that EOM dystrophy can lead to squint, ${ }^{5}$ and subsequently, Duane's retraction syndrome was found to be closely related to muscular malnutrition. ${ }^{6}$ Moreover, abnormal positions of the EOMs can also lead to strabismus. ${ }^{7}$ Additionally, dysfunction of the EOM pulleys had been shown to be involved in incomitant strabismus. ${ }^{8,9}$ Strabismus can affect the binocular visual function, resulting in impaired visual fusion in strabismus patients. ${ }^{10}$ 
Functional magnetic resonance imaging (fMRI) has been widely used to study strabismus. A previous report described reduced gray matter volume in the occipital eye field (OEF) and parietal eye field (PEF) and an increase in the gray matter volume in the frontal eye field (FEF) of adults with strabismus. ${ }^{11}$ The FEF is an area of the frontal cortex that can trigger eye movements. ${ }^{12}$ Another group demonstrated reduced functional connections between the $\mathrm{V} 1$ and $\mathrm{V} 2$ areas in macaque monkeys with strabismic amblyopia. ${ }^{13}$ It has been shown that the mean diffusivity of the occipital tracts was increased in patients with strabismic amblyopia. ${ }^{14}$

The regional homogeneity ( $\mathrm{ReHo}$ ) method, a resting-state fMRI (rs-fMRI) measurement method, is widely used to determine the local synchronization of spontaneous fMRI signals, and it provides important information on the activities of the brain. ${ }^{15,16}$ The ReHo method has been successfully deployed to investigate pathological mechanisms of neural diseases such as optic neuritis, ${ }^{17}$ sleep disorders, ${ }^{18}$ and Parkinson's disease. ${ }^{19}$ However, it has not yet been used to explore the pathophysiological changes in comitant strabismus (CS).

To our knowledge, our study is the first to evaluate regional spontaneous brain activity in subjects with CS and demonstrate its relationship with behavioral performances.

\section{Subjects and methods Subjects}

Ten men and ten women with CS were recruited from the Ophthalmology Department of the First Affiliated Hospital of Nanchang University Hospital. The diagnostic criteria for congenital strabismus included the following: 1) strabismus from birth; 2) stereopsis defects (no visual fusion); 3) binocular uncorrected or corrected visual acuity (VA) being equal; and 4) with alternated cover. The angles of the strabismus group were equal, and the large squint angle range was 50-60 delta. Subjects were excluded if they had any one of the following conditions: 1) acquired strabismus, incomitant strabismus, concealed oblique; 2) conditions of eye diseases, trauma, or optic neuropathy (eg, infection, inflammation, ischemic disease, compression damage, eye surgery, intraocular placeholder lesions); 3) psychiatric disorders, diabetes, cardiovascular disease, cerebral infarction disease; and 4) drug or alcohol addiction.

Twenty healthy controls (HCs; ten men, ten women) with similar age range, sex ratio, and education status were also recruited for this study. All HCs met the following requirements: 1) normal brain parenchyma on cranial MRI; 2) no ocular disease with uncorrected or corrected VA $>1.0 ; 3$ ) no psychiatric disease; and 4) accessible to the MRI scanning (eg, no cardiac pacemaker or implanted metal devices). This study was authorized and approved by the First Affiliated Hospital of Nanchang ethics committee. All research methods followed the Declaration of Helsinki and conformed to the principles of medical ethics. All subjects participated voluntarily and were informed of the purposes, methods, and potential risks before signing an informed consent form.

\section{MRI parameters}

MRI scanning was performed with a 3-T MR scanner (Trio, Siemens, Germany). T1-weighted images were acquired with a three-dimensional spoiled gradient-recalled sequence in an axial orientation. We obtained 176 high-resolution images taking 5 minutes (repetition time $=1,900 \mathrm{~ms}$, echo time $=2.26 \mathrm{~ms}$, thickness $=1.0 \mathrm{~mm}$, gap $=0.5 \mathrm{~mm}$, acquisition matrix $=256 \times 256$, field of view $=250 \times 250 \mathrm{~mm}$, flip angle $=9^{\circ}$ ) and 240 functional images covering the whole brain taking 10 minutes (repetition time $=2,000 \mathrm{~ms}$, echo time $=30 \mathrm{~ms}$, thickness $=4.0 \mathrm{~mm}$, gap $=1.2 \mathrm{~mm}$, acquisition matrix $=64 \times 64$, flip angle $=90^{\circ}$, field of view $=220 \times 220 \mathrm{~mm}, 30$ axial slices with gradient-recalled echo-planar imaging pulse sequence).

\section{fMRI data analysis}

The 240 functional images were analyzed as described previously. ${ }^{17}$ Briefly, the data were filtered by MRIcro (Nottingham University, Nottingham, UK) and preprocessed using SPM8 (The MathWorks, Inc., Natick, MA, US) and DPARSFA (Institute of Psychology, CAS., Beijing, People's Republic of China) software. Then, the data were processed with slice timing, head motion correction, spatial normalization, and smoothening with a Gaussian kernel of $6 \times 6 \times 6 \mathrm{~mm}^{3}$ fullwidth at half-maximum (FWHM). After spatially normalized to the Montreal Neurological Institute (MNI) space using the standard echoplanar image template and resampled at a resolution of $3 \times 3 \times 3 \mathrm{~mm}^{3}$, the fMRI images were detrended and bandpass-filtered $(0.01-0.08 \mathrm{~Hz})$ to reduce the effects of low-frequency drift and physiological high-frequency respiratory and cardiac noise.

Based on Kendall's coefficient of concordance (KCC), ReHo computation was performed with the REST software (Center for Cognition and Brain Disorders, HZNU., Hangzhou, Zhejiang, People's Republic of China), as previously described. ${ }^{17}$

\section{Statistical analysis}

To investigate the group differences in the ReHo values between patients with CS and HCs, fMRI data were fitted with a general linear model (GLM) with the SPM8 toolkit. 
We used the subject's group membership as regressors. $P<0.05$ was considered statistically significant and corrected with random field (Gaussian random field) theory with minimum $z>2.3$. Two-sample Student's $t$-test was used for continuous data for behavioral performances. All statistical analyses were done with the IBM SPSS Statistics 20.0 software (IBM Corporation, Armonk, NY, USA).

\section{Brain-behavior correlation analysis}

Brain regions with different between-group ReHo values were classified as regions of interest (ROIs) with the REST software. For each ROI, the mean ReHo value was calculated by averaging over all voxels. Finally, correlation analysis was performed to investigate the relationship between the mean ReHo value in each of those different areas and the related behavioral performances. $P<0.05$ was considered statistically significant.

\section{Clinical data analysis}

All the clinical data of the patients with CS were collected, including the course of the disease and the best-corrected VA.

\section{Results}

\section{Demographics and visual measurements}

As shown in Table 1, there were no obvious differences in weight $(P=0.453)$, age ( $P=0.705)$, best-corrected VA-right $(P=0.316)$, and best-corrected VA-left $(P=0.705)$ between the patients with CS and the HCs.

\section{ReHo differences}

Compared to HCs, patients with CS had significantly increased ReHo values in the right inferior temporal cortex (RITC)/right fusiform gyrus (RFG)/right cerebellum anterior lobe (RCAL), right lingual gyrus (RLG), and bilateral cingulate gyrus (BCG)

Table I Demographic information and clinical measures for Stra and $\mathrm{HCs}$

\begin{tabular}{lllll}
\hline Condition & Stra & HCs & t & P-values $^{\mathbf{a}}$ \\
\hline Male/female & $10 / 10$ & $10 / 10$ & $\mathrm{~N} / \mathrm{A}$ & \\
Age (years) & $29.50 \pm 9.21$ & $28.45 \pm 8.15$ & 0.382 & 0.705 \\
Weight $(\mathrm{kg})$ & $59.00 \pm 6.37$ & $58.60 \pm 5.26$ & 0.758 & 0.453 \\
Handedness & $20 \mathrm{R}$ & $20 \mathrm{R}$ & $\mathrm{N} / \mathrm{A}$ & $>0.99$ \\
Duration of strabismus & $29.50 \pm 9.21$ & $\mathrm{~N} / \mathrm{A}$ & $\mathrm{N} / \mathrm{A}$ & $\mathrm{N} / \mathrm{A}$ \\
(years) & & & & \\
Best-corrected VA-right & $\mathrm{I} .08 \pm 0.19$ & $\mathrm{I} .03 \pm 0.12$ & $\mathrm{I} .12 \mathrm{I}$ & 0.316 \\
Best-corrected VA-left & $\mathrm{I} .10 \pm 0.17$ & $\mathrm{I} .08 \pm 0.16$ & $-0.38 \mathrm{I}$ & 0.705 \\
\hline
\end{tabular}

Note: andependent Student's $t$-test.

Abbreviations: $\mathrm{HCs}$, healthy controls; N/A, not applicable; Stra, strabismus; VA, visual acuity.
(Figure 1 [red areas] and Table 1). Specifically, the mean ReHo values of altered ReHo regions between the CS group and HCs are shown in Figure 2 and Table 2.

\section{Receiver operating characteristic curve}

The differences of ReHo values in altered ReHo regions between the $\mathrm{CS}$ and $\mathrm{HC}$ groups may serve as diagnostic markers for strabismus. To test this possibility, we extracted the mean ReHo values of different brain regions and performed the receiver operating characteristic (ROC) curve analysis. In our study, the values of the area under the curve (AUC) for the RITC/RFG/RCAL (Figure 3A) and the cluster of BCG (Figure 3B) were 0.938 and 0.940 , respectively.

\section{Discussion}

To our knowledge, this is the first study to evaluate the effect of CS on resting-state brain activity using the ReHo technique. Compared to HCs, patients with CS showed significantly increased ReHo values in the RITC/RFG/RCAL, RLG, and BCG.

The human inferior temporal cortex consists of the inferior temporal gyrus, the middle temporal gyrus, and the fusiform gyrus. The inferior temporal cortex is not only responsible for visual shape selectivity but also involved in the classification of visual information. ${ }^{20,21}$ Previous studies have demonstrated that the inferior temporal gyrus responds selectively to 3D structures defined by binocular disparity. ${ }^{22,23}$ Yan et al observed that patients with comitant extropia showed decreased white matter volumes in the right inferior temporal gyrus. ${ }^{24}$ In agreement with this finding, we found significantly increased ReHo values in the RITC in patients with CS. Another complication often occurring in CS patients is the stereovision impairment. ${ }^{25}$ The ReHo values in the RITC were higher in CS patients, which may explain the compensation of stereovision in these patients.

The cerebellum is involved in balance and motor control, as well as the execution of accurate eye movements. ${ }^{26}$ Consistently, one group reported that the cerebellum is involved in the execution of eye and hand movements. ${ }^{27}$ A previous study has shown that cerebellar vermis activation is related to visually guided saccades. ${ }^{28}$ Joshi and Das $^{29}$ found that the posterior interposed nucleus (PIN) in the cerebellum plays an important role in conjugate eye movements in strabismic monkeys. In support of these findings, we also observed higher ReHo values in the cerebellum anterior lobe of patients with CS, indicating a stronger cerebellar activity than HCs. This suggests 


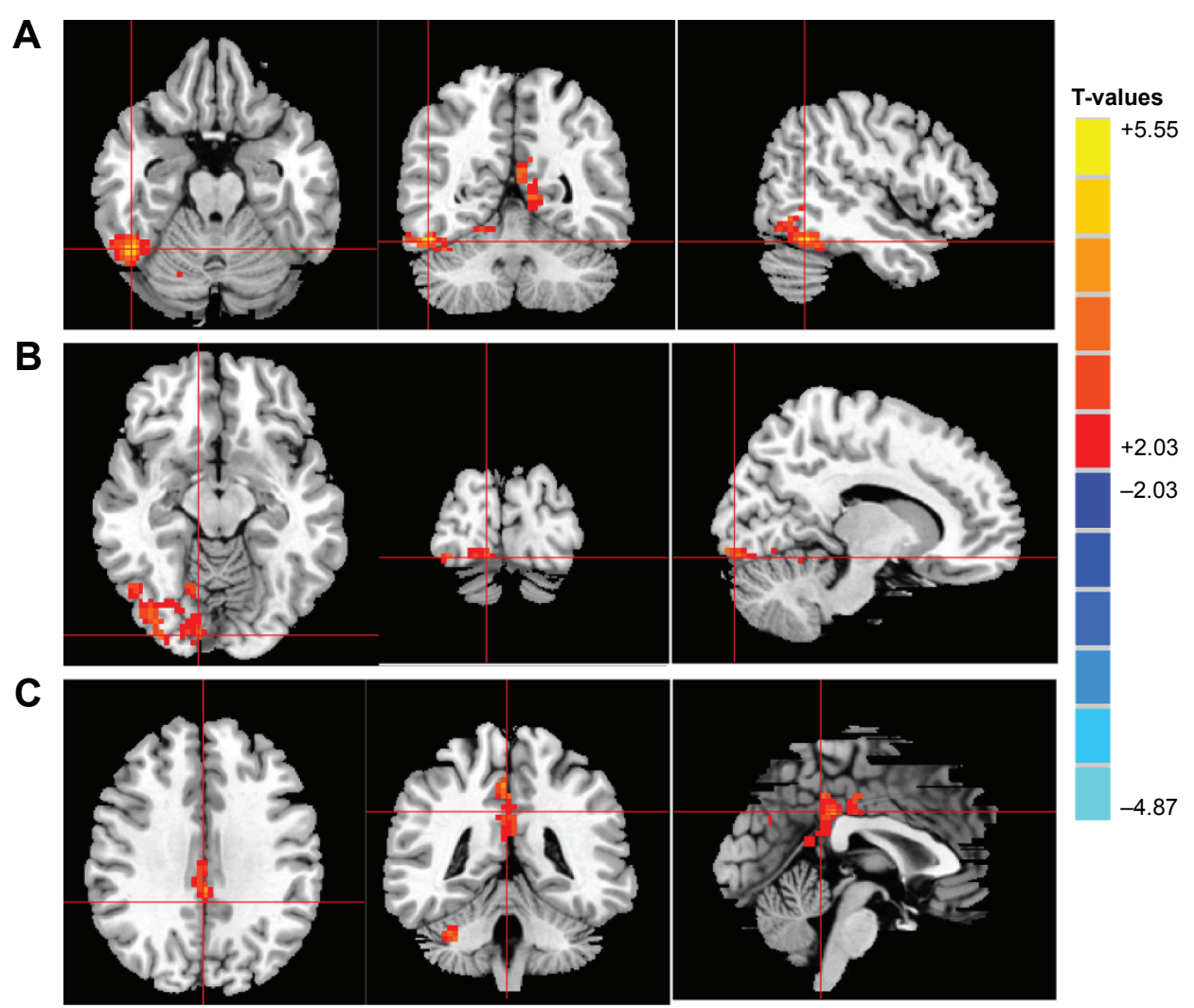

Figure I Significant differences in ReHo values between the Stra group and HCs.

Notes: The brain regions with different ReHo values are the RITC/RFG/RCAL (A), RLG (B), and BCG (C). The red areas denote higher ReHo brain regions. Multiple comparisons were performed using Gaussian random field theory $(z>2.3$, clusterwise $P<0.05$ corrected). T-values indicates the results of independent $t$-tests comparing the two groups in ReHo values in different brain regions.

Abbreviations: BCG, bilateral cingulate gyrus; HCs, healthy controls; RCAL, right cerebellum anterior lobe; ReHo, regional homogeneity; RFG, right fusiform gyrus; RITC, right inferior temporal cortex; RLG, right lingual gyrus; Stra, strabismus.

that functional reorganization may be occurring in the cerebellum anterior lobe to compensate for the conjugate eye movement abnormalities.

The lingual gyrus and fusiform gyrus are related to vision processing. ${ }^{30}$ A previous study reported that the lingual gyrus

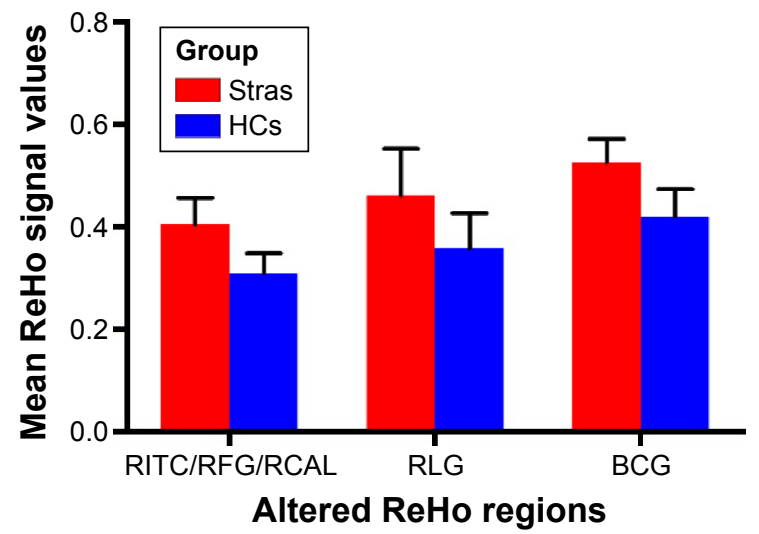

Figure 2 Mean of altered ReHo values between the Stra group and HCs. Abbreviations: BCG, bilateral cingulate gyrus; $\mathrm{HCs}$, healthy controls; RCAL, right cerebellum anterior lobe; ReHo, regional homogeneity; RFG, right fusiform gyrus; RITC, right inferior temporal cortex; RLG, right lingual gyrus; Stra, strabismus. was a stable brain region in the visual system network and that the area involved in color and visual motion (area V4) is located in the lingual and fusiform gyri of the prestriate cortex. ${ }^{31,32}$ "Visual snow" is also related to the dysfunction of lingual gyrus. ${ }^{33}$ The fusiform gyrus, a fusiform face area (FFA), is a hub for face processing. ${ }^{34}$ Yang et $\mathrm{al}^{35}$ reported that the lingual visual cortex was highly activated in patients with infantile esotropia, suggesting a potential role in optical fusion. In our study, we also found that the ReHo values in the RLG and fusiform gyrus were significantly higher, which may be related to the compensation of stereovision in CS.

The cingulate gyrus is an integral part of the limbic system, which is involved with emotion formation, depression, and pain. ${ }^{36-38}$ The limbic system is closely related to memory and emotion. ${ }^{39}$ In the present study, we also found that patients with CS had higher ReHo values in areas in the BCG, which may suggest that strabismus has a significant changes in the limbic system. Besides, the higher ReHo values in these areas may be related to compensation by the limbic system. 
Table 2 Brain regions with significantly different ReHo values between the Stra and HCs

\begin{tabular}{|c|c|c|c|c|c|c|c|}
\hline \multirow[t]{2}{*}{ ReHo, Stra $>$ HC } & \multicolumn{4}{|c|}{ Stra group and HCs } & \multicolumn{3}{|c|}{ MNI coordinates } \\
\hline & Brain areas & BA & Peak T-values & Voxels & $x$ & $y$ & $\mathbf{z}$ \\
\hline $\mathrm{a}$ & RITC/RFG/RCAL & 37 & 5.555 & 246 & 48 & -54 & -21 \\
\hline b & RLG & 18 & 3.344 & 37 & 0 & -69 & -30 \\
\hline c & BCG & 19.39 & 4.247 & 215 & 3 & -42 & 48 \\
\hline
\end{tabular}

Note: The statistical threshold was set at the voxel with $P<0.05$ for multiple comparisons using Gaussian random field theory ( $z>2.3$, clusterwise $P<0.05$ corrected). Abbreviations: BA, Brodmann area; HCs, healthy controls; MNI, Montreal Neurological Institute; Stra, strabismus; ReHo, regional homogeneity; RITC, right inferior temporal cortex; RFG, right fusiform gyrus; RCAL, right cerebellum anterior lobe; RLG, right lingual gyrus; BCG, bilateral cingulate gyrus.

\section{Conclusion}

We found that patients with CS had abnormal spontaneous activity in specific regions of the brain, which may be related to the compensation of stereovision in CS. These findings provide important information for the understanding of neural changes in CS. However, there are some limitations to our
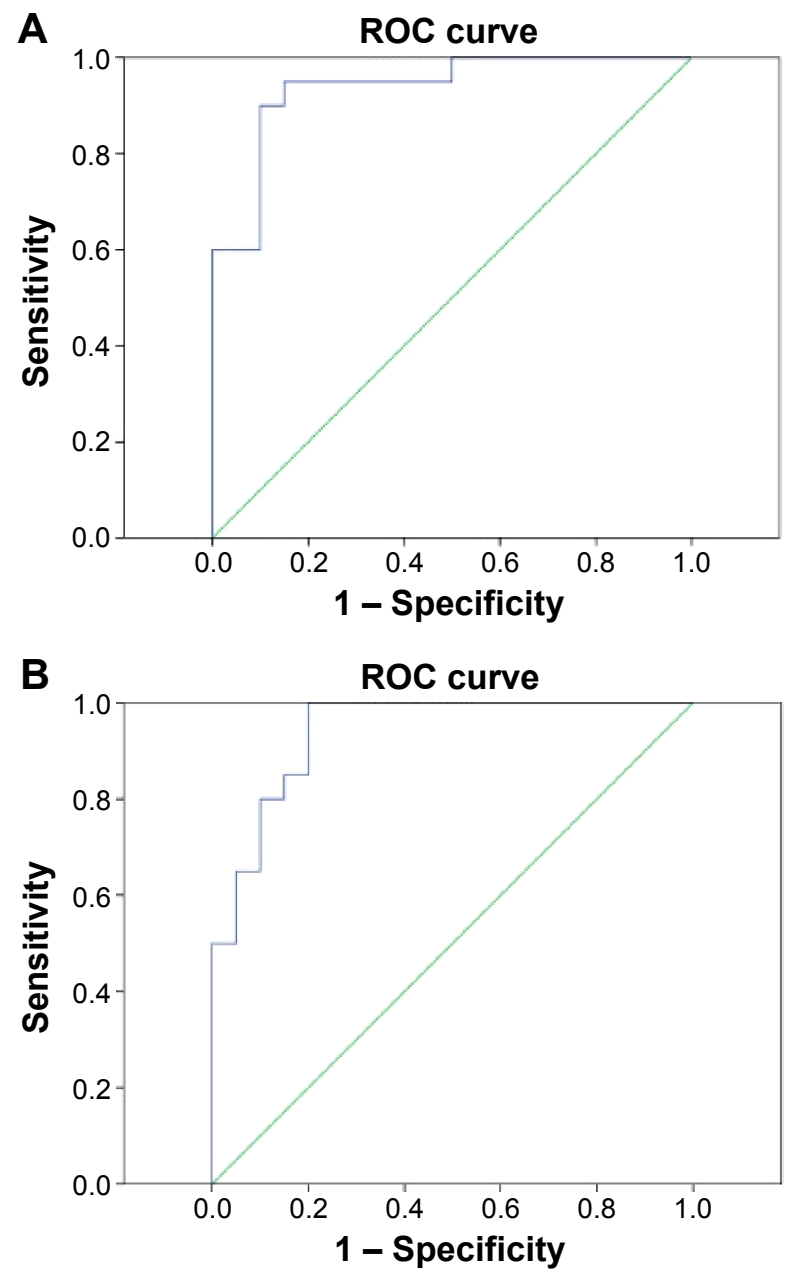

Figure 3 ROC curve analysis of the mean ReHo signal values for altered brain regions.

Notes: The area under the ROC curve was $0.938(P<0.001$; $95 \% \mathrm{Cl}: 0.864-1)$ for the RITC/RFG/RCAL (A), and $0.940(P<0.001 ; 95 \% \mathrm{Cl} 0.870-1)$ for the BCG (B). Abbreviations: $\mathrm{BCG}$, bilateral cingulate gyrus; $\mathrm{Cl}$, confidence interval; $\mathrm{RCAL}$, right cerebellum anterior lobe; ReHo, regional homogeneity; RFG, right fusiform gyrus; RITC, right inferior temporal cortex; ROC, receiver operating characteristic. study, such as the relatively small sample size. Moreover, for some subjects, the scan time was too long, so body movement could have affected the ReHo findings. Moreover, the clinical characteristics were not strict; we included both exo- and esotropic patients.

Future research should distinguish between different types of CS to more accurately assess brain function activity changes.

\section{Acknowledgments}

This study was supported by the National Natural Science Foundation of China (81160118 and 81400372); the Jiangxi Province Voyage Project (2014022); the Jiangxi Province Degree and Postgraduate Education Reform Project (2015); the Science and Technology Platform Construction Project of Jiangxi Province (2013116); the Youth Science Foundation of Jiangxi Province (20151BAB215016); the Technology and Science Foundation of Jiangxi Province (20151BBG70223); the Hunan Province Education Department Outstanding Youth Science Foundation (GJJ14170); the Health Development Planning Commission Science Foundation of Jiangxi Province (20155154); and the Scholar Project of Ganjiang River (2015).

\section{Disclosure}

The authors report no conflicts of interest in this work.

\section{References}

1. Koc F, Erten Y, Yurdakul NS. Does restoration of binocular vision make any difference in the quality of life in adult strabismus. Br J Ophthalmol. 2013;97(11):1425-1430.

2. Adams DL, Economides JR, Horton JC. Contrasting effects of strabismic amblyopia on metabolic activity in superficial and deep layers of striate cortex. J Neurophysiol. 2015;113(9):3337-3344.

3. Fu J, Li SM, Liu LR, et al. Prevalence of amblyopia and strabismus in a population of 7th-grade junior high school students in Central China: the Anyang Childhood Eye Study (ACES). Ophthalmic Epidemiol. 2014; 21(3): 197-203.

4. Simonsz HJ, Kolling GH, Unnebrink K. Final report of the early vs. late infantile strabismus surgery study (ELISSS), a controlled, prospective, multicenter study. Strabismus. 2005;13(4):169-199.

5. Lewandowski KB. Strabismus as a possible sign of subclinical muscular dystrophy predisposing to rhabdomyolysis and myoglobinuria: a study of an affected family. Can Anaesth Soc J. 1982;29(4):372-376. 
6. Honda Y, Yoshioka M. Duane's retraction syndrome associated with muscular dystrophy. J Pediatr Ophthalmol Strabismus. 1978;15(3): $157-159$.

7. Dickmann A, Petroni S, Salerni A. Effect of vertical transposition of the medial rectus muscleon primary position alignment in infantile esotropia with A- or V-pattern strabismus. J AAPOS. 2011;15(1):14-16.

8. Clark RA. The role of extraocular muscle pulleys in incomitant nonparalytic strabismus. Middle East Afr J Ophthalmol. 2015;22(3): 279-285.

9. Oh SY, Clark RA, Velez F, Rosenbaum AL, Demer JL. Incomitant strabismus associated with instability of rectus pulleys. Invest Ophthalmol Vis Sci. 2002;43(7):2169-2178.

10. Read JC. Stereo vision and strabismus. Eye (Lond). 2015;29(2): 214-224.

11. Chan ST, Tang KW, Lam KC, Chan LK, Mendola JD, Kwong KK. Neuroanatomy of adult strabismus: a voxel-based morphometric analysis of magnetic resonance structural scans. Neuroimage. 2004;22(2): 986-994.

12. Vernet M, Quentin R, Chanes L, Mitsumasu A, Valero-Cabré A. A frontal eye field, where art thou? Anatomy, function, and non-invasive manipulation of frontal regions involved in eye movements and associated cognitive operations. Front Integr Neurosci. 2014;8:66.

13. Bi H, Zhang B, Tao X, Harwerth RS, Smith EL 3rd, Chino YM. Neuronal responses in visual area V2 (V2) of macaque monkeys with strabismic amblyopia. Cereb Cortex. 2011;21(9):2033-2045.

14. Duan Y, Norcia AM, Yeatman JD, Mezer A. The structural properties of major white matter tracts in strabismic amblyopia. Invest Ophthalmol Vis Sci. 2015;56(9):5152-5160.

15. Zang Y, Jiang T, Lu Y, He Y, Tian L. Regional homogeneity approach to fMRI data analysis. Neuroimage. 2004;22(1):394-400.

16. Tononi G, McIntosh AR, Russell DP, Edelman GM. Functional clustering: identifying strongly interactive brain regions in neuroimaging data. Neuroimage. 1998;7(2):133-149.

17. Shao Y, Cai FQ, Zhong YL, et al. Altered intrinsic regional spontaneous brain activity in patients with optic neuritis: a resting-state functional magnetic resonance imaging study. Neuropsychiatr Dis Treat. 2015;11:3065-3073.

18. Dai XJ, Gong HH, Wang YX, et al. Gender differences in brain regional homogeneity of healthy subjects after normal sleep and aftersleep deprivation: a resting-state fMRI study. Sleep Med. 2012;13(6):720-727.

19. Li Y, Liang P, Jia X, Li K. Abnormal regional homogeneity in Parkinson's disease: a resting state fMRI study. Clin Radiol. 2016;71(1): e28-e34.

20. Freedman DJ, Riesenhuber M, Poggio T, Miller EK. Experiencedependent sharpening of visual shape selectivity in inferior temporal cortex. Cereb Cortex. 2006;16(11):1631-1644.

21. Sigala N. Visual categorization and the inferior temporal cortex. Behav Brain Res. 2004;149(1):1-7.

22. Verhoef BE, Vogels R, Janssen P, et al. Inferotemporal cortex subserves three-dimensional structure categorization. Neuron. 2012;73(1): $171-182$.
23. Janssen P, Vogels R, Orban GA, et al. Macaque inferior temporal neurons are selective for disparity-defined three-dimensional shapes. Proc Natl Acad Sci U S A. 1999;96(14):8217-8222.

24. Yan X, Lin X, Wang Q, et al. Dorsal visual pathway changes in patients with comitant extropia. PLoS One. 2010;5(6):e10931.

25. Serrano-Pedraza I, Manjunath V, Osunkunle O, et al. Visual suppression in intermittent exotropia during binocular alignment. Invest Ophthalmol Vis Sci. 2011;52(5):2352-2364.

26. Herzfeld DJ, Kojima Y, Soetedjo R, Shadmehr R. Encoding of action by the Purkinje cells of the cerebellum. Nature. 2015;526(7573): 439-442.

27. Nitschke MF, Arp T, Stavrou G, Erdmann C, Heide W. The cerebellum in the cerebro-cerebellar network for the control of eye and hand movements - an fMRI study. Prog Brain Res. 2005;148:151-164.

28. Hayakawa Y, Nakajima T, Takagi M, Fukuhara N, Abe H. Human cerebellar activation in relation to saccadic eye movements: a functional magnetic resonance imaging study. Ophthalmologica. 2002;216(6):399-405.

29. Joshi AC, Das VE. Muscimol inactivation of caudal fastigial nucleus and posterior interposed nucleus in monkeys with strabismus. $J \mathrm{Neu}$ rophysiol. 2013;110(8):1882-1891.

30. Bogousslavsky J, Miklossy J, Deruaz JP, Assal G, Regli F. Lingual and fusiform gyri in visual processing: a clinico-pathologic study of superior altitudinal hemianopia. J Neurol Neurosurg Psychiatry. 1987;50(5):607-614.

31. Yang YL, Deng HX, Xing GY, Xia XL, Li HF. Brain functional network connectivity based on a visual task: visual information processingrelated brain regions are significantly activated in the task state. Neural Regen Res. 2015;10(2):298-307.

32. Zeki S, Watson JD, Lueck CJ, Friston KJ, Kennard C, Frackowiak RS. A direct demonstration of functional specialization in human visual cortex. J Neurosci. 1991;11(3):641-649.

33. Schankin CJ, Maniyar FH, Sprenger T, Chou DE, Eller M, Goadsby PJ. The relation between migraine, typical migraine aura and "visual snow". Headache. 2014;54(6):957-966.

34. Ghuman AS, Brunet NM, Li Y, et al. Dynamic encoding of face information in the human fusiform gyrus. Nat Commun. 2014;5:5672.

35. Yang X, Zhang J, Lang L, Gong Q, Liu L. Assessment of cortical dysfunction in infantile esotropia using fMRI. Eur J Ophthalmol. 2014; 24(3):409-416

36. Hadland KA, Rushworth MF, Gaffan D, Passingham RE. The effect of cingulate lesions on social behaviour and emotion. Neuropsychologia. 2003;41(8):919-931.

37. Lozano AM, Mayberg HS, Giacobbe P, Hamani C, Craddock RC, Kennedy SH. Subcallosal cingulate gyrus deep brain stimulation for treatment-resistant depression. Biol Psychiatry. 2008;64(6):461-467.

38. Robin AM, Pabaney AH, Mitsias PD, Schwalb JM. Further evidence for a pain pathway involving the cingulate gyrus: a case of chronic cluster headache cured by glioblastoma. Stereotact Funct Neurosurg. 2015;93(3):194-198.

39. Lövblad KO, Schaller K, Vargas MI. The fornix and limbic system. Semin Ultrasound CT MR. 2014;35(5):459-473.
Neuropsychiatric Disease and Treatment

\section{Publish your work in this journal}

Neuropsychiatric Disease and Treatment is an international, peerreviewed journal of clinical therapeutics and pharmacology focusing on concise rapid reporting of clinical or pre-clinical studies on a range of neuropsychiatric and neurological disorders. This journal is indexed on PubMed Central, the 'PsycINFO' database and CAS,

\section{Dovepress}

and is the official journal of The International Neuropsychiatric Association (INA). The manuscript management system is completely online and includes a very quick and fair peer-review system, which is all easy to use. Visit http://www.dovepress.com/testimonials.php to read real quotes from published authors. 